\title{
The importance of being hydride
}

Adv. Energy Mater. http://doi.org/cxfg (2018)

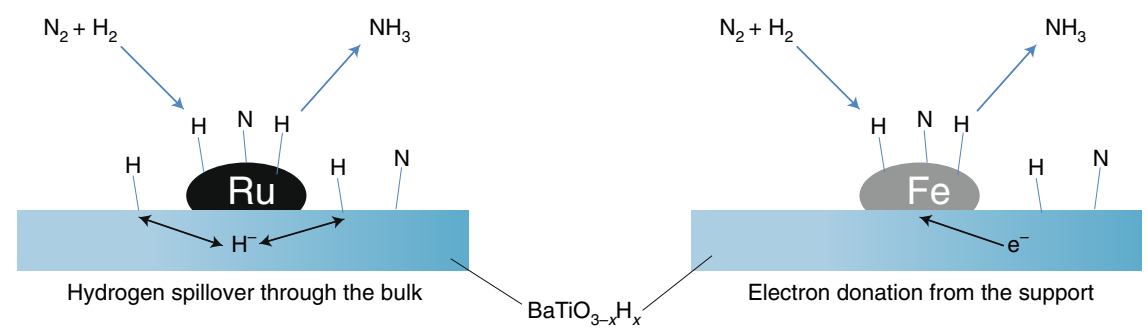

Ammonia production by the Haber-Bosch process is a century old, and yet research is still ongoing to improve its operating conditions - both a high temperature and pressure are required. Alternative catalytic systems that exploit metal hydrides or electrides as the support for ruthenium nanoparticles have been reported. However, despite the remarkable activity of such catalysts, the effect of metal hydrides on the overall system is not fully understood.

Now, Yoji Kobayashi, Hiroshi Kageyama and co-workers have shown that the introduction of hydride species into the lattice of oxide supports - $\mathrm{ATiO}_{3}$, where $\mathrm{A}=\mathrm{Ca}, \mathrm{Sr}, \mathrm{Ba}-$ has generally important effects on the activity and kinetics of the reaction; however, differences are found depending on the supported element. In a previous publication, the group had shown that barium titanate oxyhydride can activate nitrogen leading to the catalytic formation of ammonia with very competitive activity. In the present study, the team further explored this material as an active support for ruthenium and iron atoms, among others. In all cases, the presence of hydride species in the support results in a much higher activity compared with the same metal loaded on pristine barium titanate, with an activity enhancement of up to 70 times for iron. Hydrogen-deuterium exchange experiments using $\mathrm{Ru} / \mathrm{BaTiO}_{2.5} \mathrm{D}_{0.5}$ confirmed that the lattice hydrides in the support participate in the catalytic cycle - the deuterium is in fact incorporated into the ammonia.

Evaluation of the activation energy as well as reaction orders with respect to nitrogen, hydrogen and ammonia upon introduction of hydrides into the support reveals, however, important differences pointing to metal-dependent effects (pictured). In fact, the hydrogen reaction order changes from negative to positive for ruthenium, suggesting the suppression of the commonly observed hydrogen poisoning of the metal, possibly due to the hydrogen spillover ability of the support. In the case of iron, instead, the activation energy as well as the nitrogen reaction order are decreased by a factor of two, indicating a possible electron donation from the support, which facilitates the dissociation of nitrogen molecules adsorbed on iron particles.

\section{Davide Esposito}

Published online: 12 December 2018 https://doi.org/10.1038/s41929-018-0208-0 